\title{
Biochanin-A induces apoptosis and suppresses migration in FaDu human pharynx squamous carcinoma cells
}

\author{
IN-A CHO ${ }^{1}$, SANG-JOUN YOU ${ }^{2}$, KYEONG-ROK KANG ${ }^{1}$, SU-GWAN KIM ${ }^{1,6}$, \\ JI-SU OH ${ }^{1}$, JAE-SEEK YOU ${ }^{1}$, GYEONG-JE LEE ${ }^{3}$, YO-SEOB SEO ${ }^{4}$, DO KYUNG KIM ${ }^{5}$, \\ CHUN SUNG KIM ${ }^{5}$, SOOK-YOUNG LEE ${ }^{6}$ and JAE-SUNG KIM ${ }^{5}$
}

\author{
Departments of ${ }^{1}$ Oral and Maxillofacial Surgery, ${ }^{2}$ Periodontology, ${ }^{3}$ Prosthodontics, ${ }^{4}$ Oral and Maxillofacial Radiology, \\ ${ }^{5}$ Oral Biology Research Institute, ${ }^{6}$ Regional Innovation Center for Dental Science and Engineering, \\ School of Dentistry, Chosun University, Gwangju 61452, Republic of Korea
}

Received March 21, 2017; Accepted August 31, 2017

DOI: $10.3892 /$ or.2017.5953

\begin{abstract}
The aim of the present study was to investigate biochanin-A-induced anticancer effects and their cellular signaling pathway in FaDu pharyngeal squamous carcinoma cells. Biochanin-A induced cell death through increased cytotoxicity of FaDu cells in a dose- and time-dependent manner. The number of cells with nucleus condensation and the apoptotic population were increased in the $\mathrm{FaDu}$ cells stimulated with biochanin-A for $24 \mathrm{~h}$. Furthermore, extrinsic apoptotic factors such as FasL and their downstream target caspase- 8 were increased and activated in the $\mathrm{FaDu}$ cells treated with biochanin-A in a dose-dependent manner. Moreover, biochanin-A decreased the expression of intrinsic anti-apoptotic factors such as Bcl-2 and Bcl-xL, and increased the level and activation of intrinsic apoptotic factors such as Bad and caspase-9. Finally, biochanin-A induced the activation of caspase-3 and Poly(ADP ribose) polymerase (PARP) in $\mathrm{FaDu}$ cells. Our results suggest that biochanin-A-induced apoptosis was mediated by death receptor mediated-extrinsic and mitochondria-dependent intrinsic apoptotic signaling pathways. Biochanin-A also inhibited wound healing migration and proliferation of $\mathrm{FaDu}$ cells via the downregulation and inactivation of matrix metalloproteinase- 2 and -9 that are mediated by the suppression of $\mathrm{p} 38$, mitogen activated protein kinase (MAPK), NF- $\mathrm{kB}$ and Akt cellular signaling pathways. Therefore, these data suggest that the biochanin-A may act as a potential chemotherapeutic compound to treat head and neck cancer.
\end{abstract}

Correspondence to: Professor Jae-Sung Kim, Department of Pre-Dentistry, School of Dentistry, Chosun University, 309 Philmundaero, Dong-gu, Gwangju 61452, Republic of Korea

E-mail: js_kim@chosun.ac.kr

Key words: biochanin-A, pharynx squamous carcinoma, apoptosis, migration, chemotherapeutic agent

\section{Introduction}

Head and neck squamous cell carcinoma (HNSCC) is the sixth most common cancer with over 640,000 cases diagnosed annually and high mortality worldwide (1). Lifestyles such as smoking, alcohol consumption, and betel nut chewing are primary etiological factors of HNSCC and are linked to the increased prevalence of HNSCC worldwide (2). Although clinical interventions such as surgery, radiotherapy, chemotherapy, and chemo-radiotherapy have rapidly advanced, 5 -year survival and morbidity rates of patients with HNSCC have not improved significantly in the last 30 years $(2,3)$. Furthermore, despite the advancement of diagnostic techniques and therapeutics, clinical interventions frequently result in irreversible loss of function and disfigurement (4). Therefore, there is an urgent need to develop chemotherapeutic agents that effectively treat the cancer with fewer adverse effects.

Recently, natural compounds purified from herbal plants used traditionally in folk and oriental medicine have received considerable interest as a potential preventive or clinical drugs due to their various pharmacological effects combined with potential biological safety (5). Many recent studies have tested the anticancer effects of natural compounds to develop chemotherapeutic agents with fewer side-effects. Due to these studies, several natural compounds with effective anticancer effects and strong safety data have been used as clinical chemotherapeutic agents for cancer patients, after approval by the United States Food and Drug Administration (5).

Apoptosis is defined as programmed cell death characterized by morphological shrinkage (6), nucleus condensation (7) and DNA degradation (8). Cancer cell-specific apoptosis is one of current strategies for developing chemotherapeutic agents from natural compounds (9).

Biochanin-A (5,7-dihydroxy-4-methoxy-isoflavone) is a phytoestrogen, which is a natural estrogen analogue, derived from edible and herbal plants such as peanuts, alfalfa sprouts, soy and red clover (10). Recent studies have reported that biochanin-A alleviates climacteric vasomotor symptoms and reduces low-density lipoprotein levels in menopausal women (11). Furthermore, biochanin-A has been reported to 
possess various pharmacological effects including antioxidant activity (12), anti-inflammation (13), neuroprotection (14) and the prevention of articular cartilage degeneration (10). Biochanin-A has also been reported to show anticancer activity such as increased radiotoxicity in colon cancer (15), the increased survival in pancreatic cancer, and induction of apoptosis in prostate cancer (16) and hepatoma (17).

Therefore, the aim of the present study was to determine whether biochanin-A has the potential to function as a chemotherapeutic agent to treat HNSCC. We evaluated the potential apoptotic effects of biochanin-A on HNSCC and elucidated the induced apoptotic signaling pathway.

\section{Materials and methods}

Cell culture. FaDu cells originating from human pharynx squamous carcinoma were obtained from the American Type Culture Collection (ATCC; Manassas, VA, USA) and cultured by following the instruction provided by ATCC. Briefly, FaDu cells were maintained in minimum essential medium (MEM; Gibco, Grand Island, NY, USA) containing $10 \%$ fetal bovine serum (FBS; Gibco, Grand Island, NY, USA), $100 \mu \mathrm{g} / \mathrm{ml}$ streptomycin and $100 \mathrm{Unit} / \mathrm{ml}$ penicillin (Gibco) in a humidified, $5 \% \mathrm{CO}_{2}$ and $37^{\circ} \mathrm{C}$ incubator.

Cell cytotoxicity assay. To determine the cytotoxicity of biochanin-A in FaDu cells, the colorimetric MTT (3-(4,5-dimethylthiazol-2-yl)-2,5-diphenyltetrazolium bromide) assay was performed as previously described (2). Briefly, FaDu cells were cultured at a density of $0.2 \times 0^{5}$ cells $/ \mathrm{ml}$ in 96 -well plates and allowed to attach to the well overnight. After incubation, cultured FaDu cells were treated with 25, 50 and $100 \mu \mathrm{M}$ biochanin-A (Santa Cruz Biotechnology, Dallas, TX, USA) for 24 and $48 \mathrm{~h}$. Thereafter, $200 \mu \mathrm{l}$ of $5 \mathrm{mg} / \mathrm{ml} \mathrm{MTT}$ (Sigma-Aldrich, St. Louis, MO, USA) was added into cultured $\mathrm{FaDu}$ cells and incubated for another $4 \mathrm{~h}$. Sequentially, supernatant was removed, and MTT crystals were dissolved in $200 \mu \mathrm{l} /$ well dimethyl sulfoxide (DMSO; Sigma-Aldrich). Finally, optical density was measured at $570 \mathrm{~nm}$ by a spectrophotometer (Epoch; BioTek Instruments, Inc., Winooski, VT, USA).

Live and dead cell assay. To visualize the live and dead $\mathrm{FaDu}$ cells following treatment with biochanin-A, the cell live and dead assay was performed with green calcein AM and ethidium homodimer-1 as previously described (4). Briefly, FaDu cells were cultured at a density of $0.2 \times 10^{5}$ cells/ml in an 8-well chamber slide (Electron Microscopy Sciences, Hatfield, PA, USA) and allowed to attach to the well overnight. After incubation, cultured FaDu cells were treated with 25 and $50 \mu \mathrm{M}$ biochanin-A for $24 \mathrm{~h}$. Thereafter, to visualize either live or dead FaDu cells, live and dead cell assay was performed by live and dead cell assay kit (Thermo Fisher Scientific, Rockford, IL, USA), which is composed of green calcein AM to stain the live cells with green fluorescence and ethidium homodimer-1 to stain the dead cells with red fluorescence, following the instructions provided by the manufacturer. Cells were imaged using fluorescence microscopy (Eclipse TE2000; Nikon Instruments, Inc., Melville, NY, USA).
DAPI staining. To determine the nucleus condensation in FaDu cells treated with biochanin-A, DAPI (4',6-diamidino2-phenylindole) staining was performed by the protocol previously described (18). Briefly, FaDu cells were cultured at a density of $0.2 \times 10^{5}$ cells $/ \mathrm{ml}$ in an 8 -well chamber slide (Electron Microscopy Sciences) and allowed to attach to the well overnight. After incubation, cultured FaDu cells were treated with 25 and $50 \mu \mathrm{M}$ biochanin-A for $24 \mathrm{~h}$. Thereafter, $\mathrm{FaDu}$ cells were fixed with $4 \%$ paraformaldehyde after washing with phosphate-buffered solution (PBS) and stained with $1 \mathrm{mg} / \mathrm{ml}$ DAPI for $20 \mathrm{~min}$. The nucleus of FaDu cells were imaged using fluorescence microscopy (Eclipse TE2000; Nikon Instruments).

Flow cytometric analysis. Flow cytometric analysis (FACS) was performed to determine the extent of apoptosis and necrosis using Annexin V-fluorescein isothiocyanate (FITC) and propidium iodide (PI), respectively. The FaDu cells were cultured at a density of $1 \times 10^{5}$ cells $/ \mathrm{ml}$ and allowed to attach to the well overnight. After incubation, cultured FaDu cells were treated with 25 and $50 \mu \mathrm{M}$ biochanin-A for $24 \mathrm{~h}$. Thereafter, FaDu cells were washed twice in PBS and re-suspended in a binding buffer (BD Biosciences, San Diego, CA, USA). Annexin V-FITC and PI (BD Biosciences) were added to the cells and incubated in the dark for $15 \mathrm{~min}$. The cells were analyzed using a fluorescence-activated cell sorting Calibur flow cytometer (Becton-Dickinson, San Jose, CA, USA). Data analysis was performed using standard CellQuest software (Becton-Dickinson) and WinMDI version 2.9 software (The Scripps Research Institute, San Diego, CA, USA).

Immunoblotting. The FaDu cells were cultured at a density of $1 \times 10^{5}$ cells $/ \mathrm{ml}$ and allowed to attach to the well overnight. After incubation, cultured FaDu cells were treated with 25 and $50 \mu \mathrm{M}$ biochanin-A for $24 \mathrm{~h}$. Thereafter, FaDu cells were harvested, lysed using cell lysis buffer (Cell Signaling Technology, Danvers, MA, USA) containing protease inhibitor (Sigma-Aldrich) and phosphatase inhibitor cocktails (Sigma-Aldrich) according to the manufacturer's protocol. Total protein concentrations of cell lysates were determined by bicinchoninic acid (BCA) protein assays (Pierce, Rockford, IL, USA). In addition, conditioned media were harvested to detect the proteins secreted from $\mathrm{FaDu}$ cells. Equal amounts of protein and conditioned media were mixed with $5 \mathrm{X}$ loading buffer, boiled at $90^{\circ} \mathrm{C}$ for $10 \mathrm{~min}$, separated using sodium dodecyl sulfate polyacrylamide gene electrophoresis (SDS-PAGE) and transferred onto nitrocellulose membranes (BD Biosciences). After blocking with $5 \%$ bovine serum albumin (BSA; Sigma-Aldrich) in TBS-T (Tris-buffered saline with $0.1 \%$ Tween-20 (Sigma-Aldrich) at room temperature for $1 \mathrm{~h}$, membranes were reacted with TBS-T containing 5\% BSA and primary antibodies of the corresponding proteins at $4^{\circ} \mathrm{C}$ for $12 \mathrm{~h}$ and then incubated with horseradish peroxidase-conjugated secondary antibody. The following antibodies were used: antibodies against FasL, caspases (-3, -7, -8 and -9), Bcl-2, Bcl-xL, Bad, Poly(ADP ribose) polymerase (PARP), phospho-NF- $\kappa \mathrm{B}$ and total $\mathrm{NF}-\kappa \mathrm{B}$ were purchased from Cell Signaling Technology. $\beta$-actin, phospho-ERK, total ERK, phospho-p38, total p-38, phospho-Akt, total Akt, matrix metalloproteinase (MMP)-2 

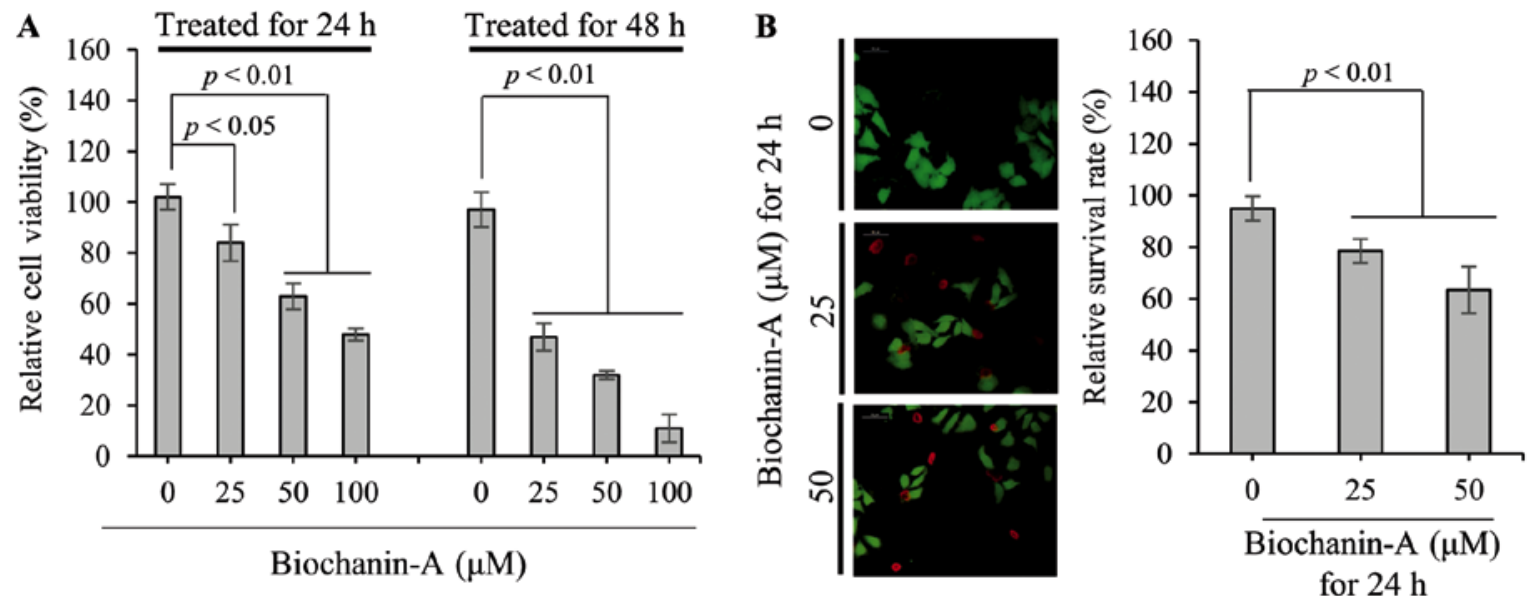

Figure 1. Biochanin-A induces cell death through the increase of cytotoxicity in FaDu cells. (A) Biochanin-A decreases the viability of FaDu cells in a dose- and time-dependent manner. FaDu cells were treated with different doses of Biochanin-A $(0,25,50$ and $100 \mu \mathrm{M})$ for 24 and $48 \mathrm{~h}$. Under the indicated treatment conditions, cell viability was measured using an MTT assay. The data represent the results of three independent experiments and are expressed as the mean \pm standard deviation ( $\mathrm{SD} ;{ }^{*} \mathrm{P}<0.05$ and ${ }^{* *} \mathrm{P}<0.01$ compared to the control). (B) Biochanin-A induces the death of FaDu cells. FaDu cells cultured at a density of $0.2 \times 10^{5}$ cells $/ \mathrm{ml}$ in 8 -well chamber slide were stimulated with 25 and $50 \mu \mathrm{M}$ biochanin-A for $24 \mathrm{~h}$. Live cells were stained with green calcein-AM and dead cells were stained with ethidium homodimer-1 (red) and then examined and photographed using fluorescence microscopy (Eclipse TE200; Nikon Instruments).

and MMP-9 were purchased from Santa Cruz Biotechnology. The immunoreactive bands were visualized using the ECL system (Amersham Biosciences, Piscataway, NJ, USA) and were exposed on radiographic film.

In vitro migration assay. To perform the migration assay, FaDu cells were cultured onto $2 \times 0.22 \mathrm{~cm}^{2}$ culture inserts (Ibidi, Regensburg, Germany) at a density of $1 \times 10^{4}$ cells/well. Wounds were introduced by removing the culture inserts after $24 \mathrm{~h}$ of incubation. Thereafter, cultured FaDu cells were treated with $25 \mu \mathrm{M}$ biochanin-A for 48 and $72 \mathrm{~h}$. Wound widths were imaged using an inverted microscope (Eclipse TE2000; Nikon Instruments).

Colony formation assay. A colony formation assay was performed according to the previously described protocol (19). Briefly, FaDu cells were cultured at a density of 200 cells/well in a 6-well culture plate and allowed to attach to the well overnight. After incubation, cultured FaDu cells were treated with 25 and $50 \mu \mathrm{M}$ biochanin-A for $24 \mathrm{~h}$ and were then incubated in culture medium without biochanin-A for 5 days. Thereafter, the medium was removed and the cells were washed with PBS and fixed with $4 \%$ paraformaldehyde for 10 min at $4^{\circ} \mathrm{C}$. Sequentially, the colonies were stained with $2 \%$ crystal violet for $10 \mathrm{~min}$. Finally, the colonies were washed with PBS and dried at room temperature, before being imaged by a digital camera (Nikon Instruments).

Gelatin zymography. Gelatin zymography was performed to assess the activity of matrix metalloproteinases (MMPs) secreted from FaDu cells treated with biochanin-A. An equal volume of conditioned media was mixed with non-reducing sample buffer [4\% SDS, $0.15 \mathrm{M}$ Tris ( $\mathrm{pH} 6.8)$ and $20 \%(\mathrm{v} / \mathrm{v})$ glycerol containing $0.05 \%(\mathrm{w} / \mathrm{v})$ bromophenol blue] and resolved on a $10 \%$ polyacrylamide gel containing copolymerized $0.2 \%(1 \mathrm{mg} / \mathrm{ml})$ swine skin gelatin. After electrophoresis of the conditioned media samples, gels were washed with cold PBS containing 2.5\% (v/v) Triton X-100 for $30 \mathrm{~min}$ and washed twice with cold PBS for $15 \mathrm{~min}$. After washing, gels were incubated in the zymogram renaturing buffer $[50 \mathrm{mM}$ Tris- $\mathrm{HCl}$ (pH 7.6), $10 \mathrm{mM} \mathrm{CaCl}_{2}, 50 \mathrm{mM} \mathrm{NaCl}$ and $0.05 \%$ Brij-35) at $37^{\circ} \mathrm{C}$ for $72 \mathrm{~h}$. After renaturation of MMPs, gels were stained with $0.1 \%$ Coomassie brilliant blue R250. The gelatinolytic activity was revealed as a clear band on a background of uniform light blue staining.

Statistical analysis. Data are reported as the mean \pm SD of three individual experiments performed. Statistical analysis was carried out using the Student's t-test and a P-value $<0.05$ was considered to indicate a statistically significant result.

\section{Results}

Biochanin-A induces death of FaDu cells via increased cytotoxicity. To measure the viability of FaDu cells treated with 25,50 and $100 \mu \mathrm{M}$ biochanin-A for 24 and $48 \mathrm{~h}$, MTT assays were performed. As shown in Fig. 1A, relative viabilities of FaDu cells treated with 25,50 and $100 \mu \mathrm{M}$ biochanin-A for $24 \mathrm{~h}$ are $84 \pm 7.2,63 \pm 5.15$ and $48 \pm 2.4 \%$, respectively, compared to non-treated control $(102 \pm 5.1 \%)$. Furthermore, relative viabilities of $\mathrm{FaDu}$ cells treated with the same concentrations of biochanin-A for $48 \mathrm{~h}$ are $47 \pm 5.3$, $32 \pm 1.6$ and $11 \pm 5.5 \%$, respectively. Live and dead cell assays were performed to visualize the live and dead cells after treatment with 25 and $50 \mu \mathrm{M}$ biochanin-A for $24 \mathrm{~h}$. In the non-treated control, as shown in Fig. 1B, almost all cells are stained fluorescent green by membrane permeable calcein $\mathrm{AM}$, which is cleaved by cytosolic esterase in living cells. In contrast, the number of dead cells stained fluorescent red by ethidium homodimer-1 increases with biochanin-A treatment in a dose-dependent manner. The relative rate of live FaDu cells after treatment with 25 and $50 \mu \mathrm{M}$ biochanin-A were measured as $78.5 \pm 4.7$ and $63.5 \pm 9 \%$, respectively. These results consistently demonstrate that biochanin-A suppresses 
$\mathbf{A}$

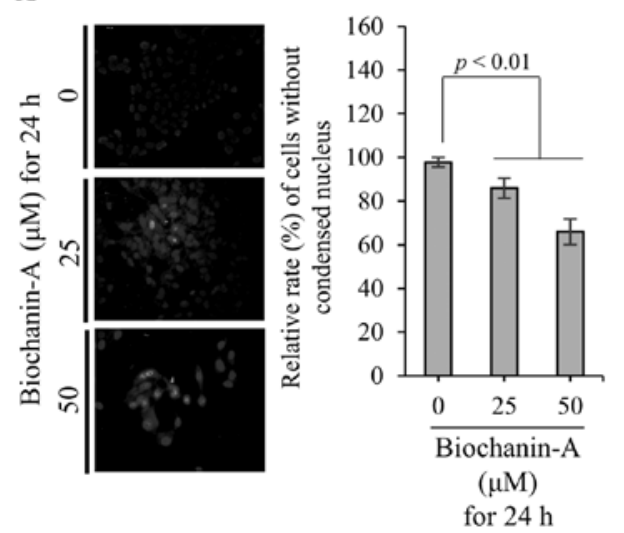

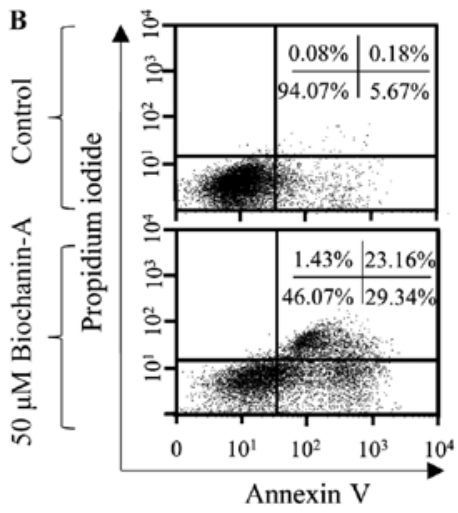

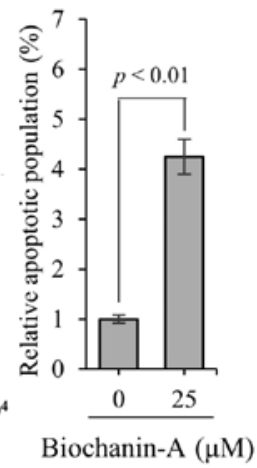

Figure 2. Biochanin-A-induced FaDu cell death is mediated by apoptosis. (A) Biochanin-A increases FaDu cells with condensed nucleus in a dose-dependent manner. Detection of apoptotic cells was accomplished by fluorescently staining DNA to examine chromosomal condensation. FaDu cells cultured at a density of $1 \times 10^{5}$ cells $/ \mathrm{ml}$ were treated with 0,25 and $50 \mu \mathrm{M}$ biochanin-A and incubated for $24 \mathrm{~h}$. Cells were stained with 4'-6-diamidino-2-phenylindole (DAPI) and then examined and photographed using fluorescence microscopy. (B) Increased apoptotic population in FaDu cells treated with biochanin-A. Flow cytometric analysis was performed on cells co-stained with Annexin V-FITC and propidium iodide (PI) (Cell Signaling Technology) to detect apoptosis. After 5x10 cells $/ \mathrm{ml}$ of FaDu cells were plated into a 6-well plate. After $24 \mathrm{~h}$, the cells were treated with $50 \mu \mathrm{M}$ biochanin-A. Both floating and attached cells were then collected, washed twice with ice-cold phosphate-buffered saline, and resuspended in $500 \mu 1$ of $1 \mathrm{X}$ binding buffer. Annexin V-FITC and PI were added to the cells for $15 \mathrm{~min}$ at $37^{\circ} \mathrm{C}$ in the dark. The population of Annexin V-positive cells and the cell cycle phase were analyzed using a BD CellQuest ${ }^{\circledR}$ version 3.3 instrument (Becton-Dickinson) and WinMDI version 2.9 software (The Scripps Research Institute).

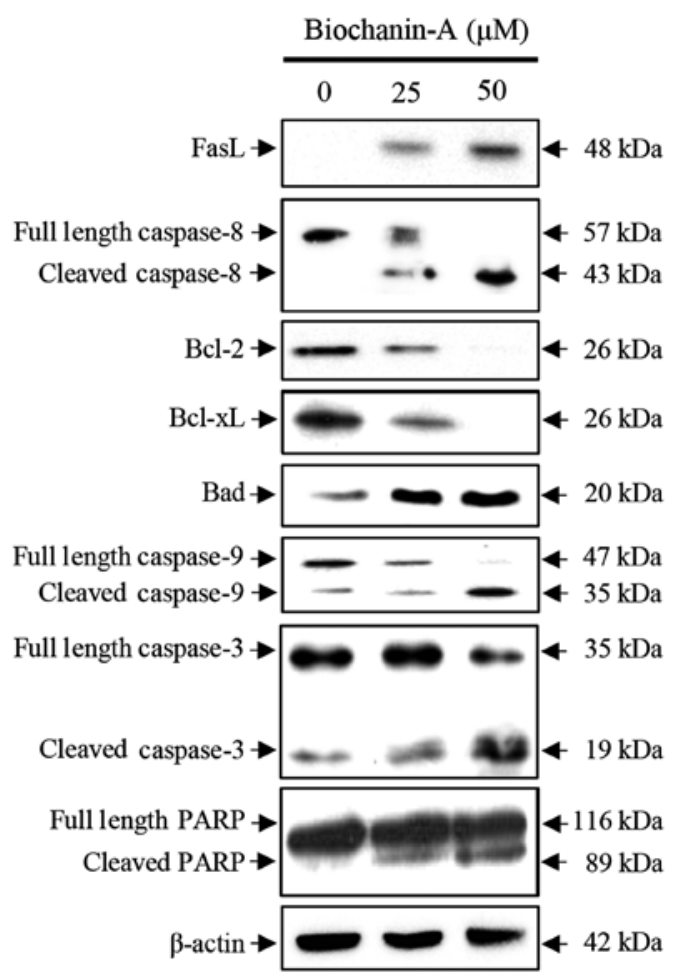

Figure 3. Biochanin-A-induced apoptosis is mediated by extrinsic and intrinsic apoptotic pathways. FaDu cells cultured at a density of $1 \times 10^{5}$ cells $/ \mathrm{ml}$ in 6 -well plates were stimulated with 25 and $50 \mu \mathrm{M}$ biochanin-A for $24 \mathrm{~h}$. The activation of caspase-3 and PARP via biochanin-A induced extrinsic death receptor-dependent and intrinsic mitochondrial-dependent apoptosis signaling pathways. Biochanin-A -induced apoptosis of FaDu cells is dependent on caspase activation.

the viability of FaDu cells through an increase in cell cytotoxicity, in a dose- and time-dependent manner.

Biochanin-A induced FaDu cell death is mediated by apoptosis. Nucleus condensation is a typical phenomenon of apoptosis (7). Therefore, DAPI staining was performed to observe the nucleus morphology of FaDu cells treated with biochanin-A. As shown in Fig. 2A, the relative rate of $\mathrm{FaDu}$ cells without condensed nucleus is $\sim 85.9 \pm 4.7$ and $66.0 \pm 5.9 \%$ at 25 and $50 \mu \mathrm{M}$ biochanin-A compared with non-treated control, respectively. These data show that biochanin-A increased the number of FaDu cells with condensed nuclei in a dose-dependent manner. Therefore, to verify the biochanin-A-induced apoptosis of FaDu cells, FACS analysis was performed using Annexin V-FITC and PI. As shown in Fig. 2B, the apoptotic population of $\mathrm{FaDu}$ cells treated with $50 \mu \mathrm{M}$ biochanin-A increases $\sim 4$.2-fold compared with than non-treated control. Taken together, these data consistently indicate that biochaninA-induced $\mathrm{FaDu}$ cell death is mediated by apoptosis.

Biochanin-A induces the apoptosis of FaDu cell through extrinsic and intrinsic apoptotic pathways. Next, to verify biochanin-A-induced apoptosis in FaDu cells, the alteration in expression of pro- and anti-apoptotic factors were observed by western blot analysis. As shown in Fig. 3, the expression of death ligand FasL (48 kDa) increases in FaDu cells treated with 25 and $50 \mu \mathrm{M}$ biochanin-A in a dose-dependent manner. Sequentially, the expression of full length caspase-8 $(57 \mathrm{kDa})$, which is a down-stream target molecule of FasL and is part of the death receptor mediated extrinsic apoptotic signaling pathway, gradually decreased in the FaDu cells treated with biochanin-A. In contrast, the expression of cleaved caspase- 8 ( $43 \mathrm{kDa})$ increased due to the cleavage of full length caspase- 8 by FasL in the FaDu cells treated with biochanin-A. Furthermore, the expression of cleaved caspase- 3 increased due to the cleavage of full length caspase-3 (35 kDa) by the cleaved caspase- 8 . Sequentially, the expression of cleaved PARP ( $89 \mathrm{kDa}$; full length PARP: $116 \mathrm{kDa}$ ) increased by cleaved caspase- 3 and then induced apoptosis. Therefore, these data indicate that the biochanin-A induces cells death through the FasL-caspase-8-caspase-3- 

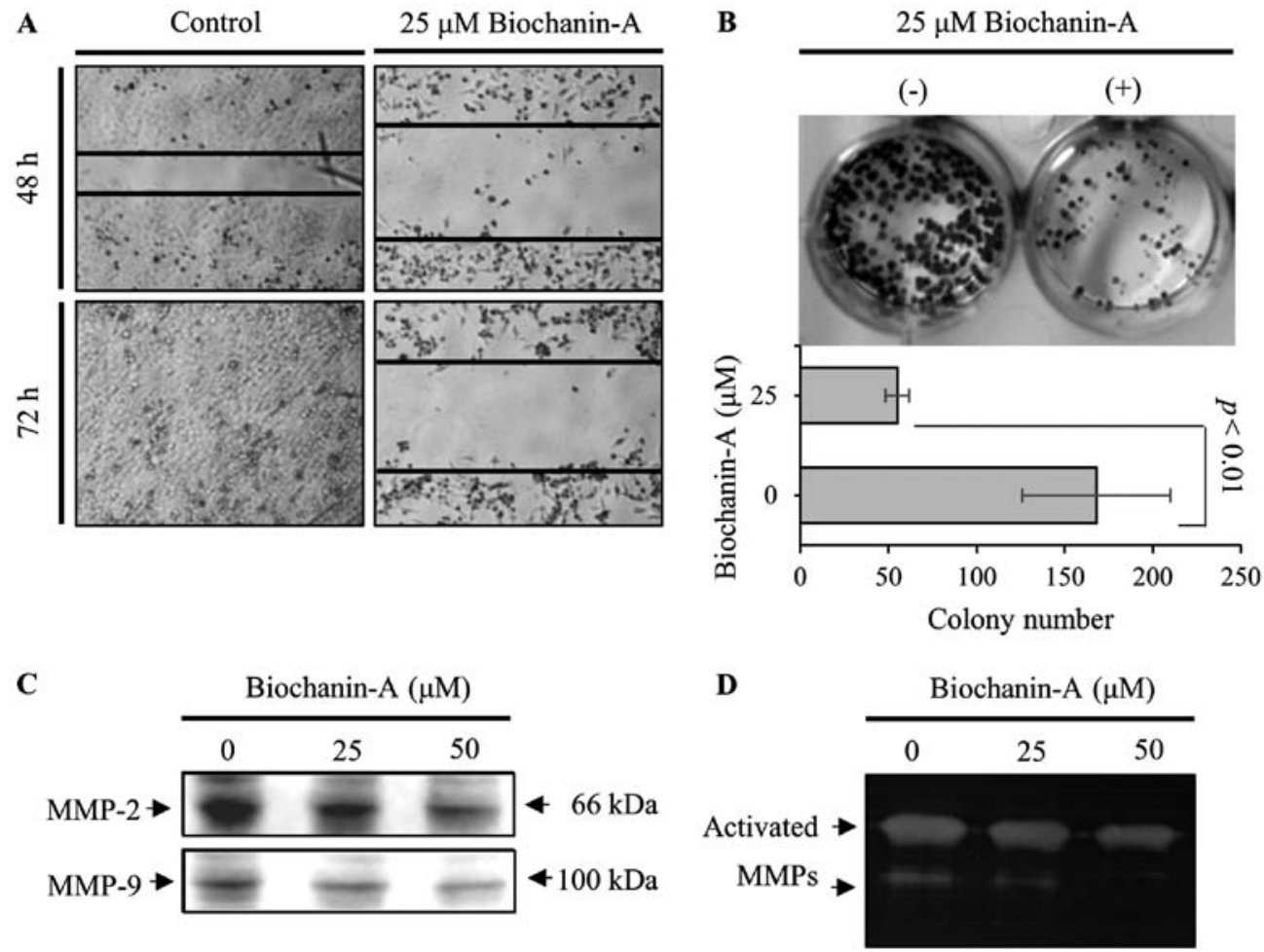

Figure 4. Biochanin-A suppresses the migration and proliferation of FaDu cells through downregulation and inactivation of matrix metalloproteinase (MMP)-2 and -9. (A) Wound healing migration is attenuated in FaDu cells treated with biochanin-A. FaDu cells were treated with $25 \mu \mathrm{M}$ biochanin-A for 48 and $72 \mathrm{~h}$. Wound widths were imaged using reverse microscopy (Eclipse TE2000; Nikon Instruments). (B) Biochanin-A suppresses the proliferation of FaDu cells. To perform a colony formation assay, FaDu cells were treated with $25 \mu \mathrm{M}$ biochanin-A for $24 \mathrm{~h}$ and then were incubated in the culture media without biochanin-A for 5 days. Thereafter, formed colonies were stained by crystal violet and were counted. (C and D) The expressions and activities of MMP-2 and MMP-9 decreased in FaDu cells treated with biochanin-A in a dose-dependent manner. FaDu cells were stimulated with 25 and $50 \mu \mathrm{M}$ biochanin-A for $24 \mathrm{~h}$. Thereafter, (C) immunoblotting and (D) gelatin zymography were performed to verify the expression and activity of MMP-2 and MMP-9 in the conditioned media of FaDu cells treated with biochanin-A, respectively.

PARP axis mediated extrinsic apoptosis signaling pathway in FaDu cells.

Furthermore, the expressions of Bcl-2 (26 kDa) and Bcl-xL $(26 \mathrm{kDa})$, anti-apoptotic factors associated with the mitochondria-dependent apoptotic signaling pathway, decreased in the FaDu cells treated with 25 and $50 \mu \mathrm{M}$ biochanin-A in a dose-dependent manner. Whereas, biochanin-A increased the expression of Bad (20 kDa) and cleaved caspase-9 (35 kDa; full length caspase-9: $47 \mathrm{kDa}$ ), pro-apoptotic factors associated with the mitochondria-dependent apoptotic signaling pathway. The cleaved caspase- 9 increased the amount of cleaved caspase- 3 and cleaved PARP and then induced the apoptosis of FaDu cells treated with biochanin-A. Therefore, these data indicate that biochanin-A induces cell death via the mitochondria-dependent intrinsic apoptosis signaling pathway in $\mathrm{FaDu}$ cells.

Biochanin-A suppresses the migration and proliferation of $\mathrm{FaDu}$ cells through the downregulation and inactivation of $M M P-2$ and -9 . FaDu cells were treated with $25 \mu \mathrm{M}$ biochaninA for 48 and $72 \mathrm{~h}$ to verify whether biochanin-A can suppress the migration and invasion. As shown in Fig. 4A, biochanin-A effectively suppressed migration compared with non-treated control. In addition, to verify whether biochanin-A suppress the proliferation of $\mathrm{FaDu}$ cells, a colony formation assay was performed as shown in Fig. 4B. The number of colonies in the untreated control was $168 \pm 42$. In contrast, the number of colonies was $55 \pm 6.6$ in the $\mathrm{FaDu}$ cells treated with $25 \mu \mathrm{M}$ biochanin-A for 5 days. These data indicate that biochanin-A effectively suppressed the migration and proliferation of $\mathrm{FaDu}$ cells. In order to verify the expression alteration of implicating factors associated with the migration and proliferation of cancer cells, western blot analysis and gelatin zymography were performed as shown as Fig. 4C and D, respectively. The expressions of MMP-2 and MMP-9 gradually decreased in the FaDu cells treated with 25 and $50 \mu \mathrm{M}$ biochanin-A in a dose-dependent manner. Clear bands formed on the gelatin zymogram gels by active MMPs gradually decreased in the FaDu cells treated with 25 and $50 \mu \mathrm{M}$ biochanin-A in a dose-dependent manner. These data consistently indicate that biochanin-A suppresses the migration and invasion of $\mathrm{FaDu}$ cells through the downregulation expression and activation of MMPs. Taken together, these data suggest that the biochaninA may act as a potential anti-metastatic reagent in HNSCC.

To verify the cellular signaling associated with migration and proliferation of FaDu cells, alterations in ERK1/2, p38 and NF- $\mathrm{KB}$ expression were observed by immunoblotting in $\mathrm{FaDu}$ cells treated with $50 \mu \mathrm{M}$ biochanin-A for 15,30 and $60 \mathrm{~min}$ as shown in Fig. 5. Biochanin-A did not induce the phosphorylation of ERK1/2 in FaDu cells at the defined treatment time, whereas, the phosphorylation of $\mathrm{p} 38$ and NF- $\mathrm{kB}$ decreased in the FaDu cells treated with biochanin-A in a time-dependent manner compared with non-treated control. Furthermore, the phosphorylation of Akt, which is closely associated with 


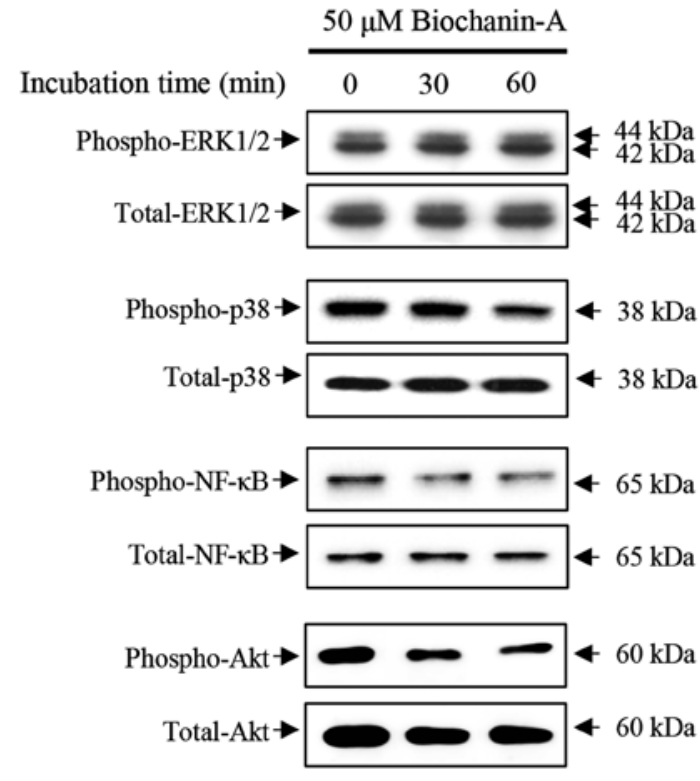

Figure 5. Biochanin-A suppresses the migration and proliferation of $\mathrm{FaDu}$ cells through the inhibition of $\mathrm{p} 38 \mathrm{MAPK}, \mathrm{NF}-\mathrm{kB}$ and Akt phosphorylation. FaDu cells cultured at a density of $1 \times 10^{5}$ cells $/ \mathrm{ml}$ in 6 -well plates were stimulated with $50 \mu \mathrm{M}$ biochanin-A for 30 and $60 \mathrm{~min}$. Thereafter, FaDu cells were harvested and lysed using cell lysis buffer containing the phosphatase inhibitors. Immunoblotting was performed to verify the alterations of ERK1/2, $\mathrm{p} 38, \mathrm{NF}-\mathrm{\kappa B}$ and Akt. The immunoreactive bands were visualized using the ECL system and were exposed on radiographic film.

cellular signaling of cell proliferation, significantly decreased in $\mathrm{FaDu}$ cells treated with biochanin-A, in a time-dependent manner compared with non-treated control. Therefore, these data suggest that biochanin-A induced suppression of migration and proliferation is associated with the alteration of $\mathrm{p} 38$ MAPK, NF- $\mathrm{KB}$ and PI3K/Akt cellular signaling pathways in FaDu cells.

\section{Discussion}

Oral cancer is the most common type of head and neck cancer and head and neck squamous cell carcinomas worldwide (20). Despite therapeutic and technological advances, the 5-year survival rate of oral cancer has not increased (21). Furthermore, the clinical interventions for patients with oral cancer have multiple serious side-effects such as the loss of function of speech, breathing, mastication and swallowing (22). Therefore, to reduce the side-effects caused by clinical interventions to treat oral cancer, the development of chemotherapy using natural compounds that possess anticancer properties and excellent biological safety is critical.

Phytoestrogens are plant-derived compounds that are structurally and physiologically similar to estrogen. Recent studies have reported that the phytoestrogens such as coumestrol, kaempferol and genistein induce apoptosis in various cancers including breast, prostate and hepatocellular carcinoma (23-25).

Biochanin-A is a phytoestrogen derived from red clover and used as a folk medicine worldwide. In the present study, we demonstrated that biochanin-A induced anticancer activities including the suppression of cellular proliferation and migration, and the increase in apoptosis through the induction of caspase activation in FaDu head and neck squamous cell carcinoma.

In our previous study, we reported that biochanin-A did not affect the viability of primary chondrocytes isolated from the articular cartilage of rats for 21 days (10). However, the present study showed that biochanin-A increased cell death through the increase of cytotoxicity in FaDu cells, as shown in Fig. 1. Furthermore, recent studies have reported that biochanin-A increased the cytotoxicity of pancreatic (26) and prostate cancer (16). Therefore, these studies suggest that biochanin-A is a phytoestrogen that induce specific cancer cell death.

DNA fragmentation caused by nucleus condensation is a typical phenomenon of apoptosis, which is a programmed cell death. As shown in Fig. 2A, the number of FaDu cells with condensed nucleus was increased by biochanin-A in a dose-dependent manner. Therefore, these data indicate that biochanin-A-induced FaDu cell death may involve apoptosis. In order to verify this, FACS analysis using Annexin V and PI staining was performed, as shown in Fig. 2B. At the early stage of apoptosis, membrane phosphatidylserine is translocated to the cell surface from the inner plasma membrane. Therefore, Annexin $\mathrm{V}$, which is a $\mathrm{Ca}^{2+}$-dependent phospholipid-binding protein with binding activity to phosphatidylserine, was used as a marker to detecting the early stage of apoptosis (21). Furthermore, translocated membrane phosphatidylserine precedes the loss of membrane integration. Subsequently, PI without membrane permeability binds to DNA by intercalating between the bases through the membranes of dead and damaged cells in the late stage of apoptosis (21). In this study, the results of FACS analysis showed that the biochanin-A increased the apoptotic population of FaDu cells in both early and late stage compared with non-treated control. Taken together, these data consistently indicate that biochanin-A induces apoptosis in $\mathrm{FaDu}$ cells.

Apoptosis is generally divided into the death receptor mediated extrinsic pathway and mitochondrial-dependent intrinsic pathway $(27,28)$. The death receptor mediated extrinsic pathways are triggered by death ligands such as FasL and TNF-related apoptosis-inducing ligand (TRAIL), and are sequentially mediated by the activation of caspase- 8 , caspase-3, and PARP to induce DNA fragmentation $(27,28)$. However, the mitochondria-dependent intrinsic pathway associated with apoptosis is triggered by growth hormone withdrawal, DNA damage by UV- or $\gamma$-radiation, chemotherapeutic drugs, and the activation of caspase- 8 associated death receptor mediated extrinsic apoptosis through the upregulation or activation of pro-apoptotic factors such as Bad, Bid, Bax and caspase- 9 and the downregulation of anti-apoptotic factors such as Bcl-2 and Bcl-xL $(27,28)$. Finally, activated caspase-9 induces the activation of caspase- 3 and PARP to induce DNA fragmentation $(27,28)$. In the present study, biochanin-A increased the expression of FasL and induced the activation of its downstream pro-apoptotic factor caspase- 8 as shown in Fig. 3. Furthermore, the expression of anti-apoptotic factors such as Bcl-2 and Bcl-xL dose-dependently decreased in the $\mathrm{FaDu}$ cells treated with biochanin-A. Whereas, biochanin-A increased the expression of Bad and activated caspase-9 in FaDu cells. Finally, both activated caspase- 8 and caspase- 9 increased the activation of caspase- 3 and PARP to induce the apoptosis of FaDu cells treated with biochanin-A. Therefore, 
these data indicate that biochanin-A-induced FaDu cell death is mediated by both death receptor mediated extrinsic and mitochondria dependent intrinsic apoptosis pathways.

Next, in the present study, we demonstrated that the migration and proliferation of $\mathrm{FaDu}$ cells were significantly decelerated by biochanin-A as shown in Fig. 4A and B. Cancer cells metastasize to other tissues through bloodstream and lymphatic system in the terminal stage of cancer (29). Hence, the deceleration of cancer cell growth and the suppression of metastasis are crucial aims of clinical treatment for metastatic cancer (30). Furthermore, the expressional upregulation and activation of matrix metalloproteinase such as MMP-2 and MMP-9 are crucial factors associated with the migration and proliferation of cancer cells $(29,31)$. We demonstrated that the expression and activation of MMP-2 and MMP-9 significantly decreased in the FaDu cells treated with biochanin-A, as shown in Fig. 4C and D. Taken together, biochanin-A decelerates the migration and proliferation of FaDu cells through the expression downregulation and inactivation of MMP-2 and MMP-9.

In the present study, biochanin-A suppressed the phosphorylation of p38 MAPK and NF- $\mathrm{B}$ in FaDu cells as shown in Fig. 5. As well as this study, Kole et al (32), reported that biochanin-A-induced anti-proliferation through the inhibition of p38 MAPK and the blocking of NF- $\kappa$ B nuclear translocation. Recent studies have also reported that the expression of MMP-2 and MMP-9, associated with cell growth, proliferation and migration, are closely regulated by MAPK cellular signaling in various cancer cells including colon cancer (33) and osteosarcoma (34). Furthermore, Bindhu et al (35) and Qin et al (36) reported that the activation of MMP-2 is closely regulated by $\mathrm{NF}-\kappa \mathrm{B}$ cellular signaling in oral squamous cell carcinoma and breast cancer. These consistently indicate that biochanin-A-induced anti-proliferation and anti-migration are mediated by the expressional downregulation and inactivation of MMP-2 and MMP-9 through the suppression of p38 MAPK and NF- $\kappa$ B cellular signaling pathways in FaDu cells. In addition, PI3K/Akt signaling is closely associated with cell proliferation, survival, growth and metastasis $(37,38)$. Recent studies have reported that the suppression of PI3K/Akt signaling enhances cytotoxicity through the inhibition of the mTOR signaling pathway in various cancers such as breast and colorectal cancer (39-41). In the present study, we demonstrated that biochanin-A suppressed the phosphorylation of Akt in FaDu cells. Taken together, these studies indicate that biochanin-A-induced anticancer activities including apoptosis, anti-proliferation, and anti-migration may be mediated by the suppression of PI3K/Akt cellular signaling pathway in $\mathrm{FaDu}$ cells.

In conclusion, we demonstrated that biochanin-A-induced anti-apoptotic effects such as death receptor mediated extrinsic and mitochondria-dependent intrinsic apoptosis, anti-metastasis and anti-proliferation in FaDu cells. These findings suggest that biochanin-A may be a promising natural chemotherapy candidate for HNSCC treatment.

\section{Acknowledgements}

The present study was supported by a research fund from the Chosun University Dental Hospital, 2016.

\section{References}

1. Lacko M, Braakhuis BJ, Sturgis EM, Boedeker CC, Suárez C, Rinaldo A, Ferlito A and Takes RP: Genetic susceptibility to head and neck squamous cell carcinoma. Int J Radiat Oncol Biol Phys 89: 38-48, 2014.

2. Park MR, Kim SG, Cho IA, Oh D, Kang KR, Lee SY, Moon SM, Cho SS, Yoon G, Kim CS, et al: Licochalcone-A induces intrinsic and extrinsic apoptosis via ERK1/2 and p38 phosphorylation-mediated TRAIL expression in head and neck squamous carcinoma FaDu cells. Food Chem Toxicol 77: 34-43, 2015.

3. Vigneswaran N, Wu J, Song A, Annapragada A and Zacharias W: Hypoxia-induced autophagic response is associated with aggressive phenotype and elevated incidence of metastasis in orthotopic immunocompetent murine models of head and neck squamous cell carcinomas (HNSCC). Exp Mol Pathol 90: 215-225, 2011.

4. Kim JS, Oh D, Yim MJ, Park JJ, Kang KR, Cho IA, Moon SM, Oh JS, You JS, Kim CS, et al: Berberine induces FasL-related apoptosis through p38 activation in KB human oral cancer cells. Oncol Rep 33: 1775-1782, 2015.

5. Kinghorn AD, Pan L, Fletcher JN and Chai H: The relevance of higher plants in lead compound discovery programs. J Nat Prod 74: 1539-1555, 2011.

6. McCarthy JV and Cotter TG: Cell shrinkage and apoptosis: A role for potassium and sodium ion efflux. Cell Death Differ 4: 756-770, 1997.

7. Fang M, Zhang HQ and Xue SB: Apoptosis of HL-60 cells induced by Harringtonine: Membrane blebs, nucleus blebs and chromatin condensation. Shi Yan Sheng Wu Xue Bao 29: 221-233, 1996 (In Chinese).

8. Nagata S, Nagase H, Kawane K, Mukae N and Fukuyama H: Degradation of chromosomal DNA during apoptosis. Cell Death Differ 10: 108-116, 2003.

9. Fesik SW: Promoting apoptosis as a strategy for cancer drug discovery. Nat Rev Cancer 5: 876-885, 2005.

10. Oh JS, Cho IA, Kang KR, You JS, Yu SJ, Lee GJ, Seo YS, Kim CS, Kim K, Kim SG, et al: Biochanin-A antagonizes the interleukin$1 \beta$-induced catabolic inflammation through the modulation of $\mathrm{NF}-\kappa \mathrm{B}$ cellular signaling in primary rat chondrocytes. Biochem Biophys Res Commun 477: 723-730, 2016.

11. Booth NL, Piersen CE, Banuvar S, Geller SE, Shulman LP and Farnsworth NR: Clinical studies of red clover (Trifolium pratense) dietary supplements in menopause: A literature review. Menopause 13: 251-264, 2006.

12. Guo Q, Rimbach G, Moini H, Weber S and Packer L: ESR and cell culture studies on free radical-scavenging and antioxidant activities of isoflavonoids. Toxicology 179: 171-180, 2002.

13. Ming X, Ding M, Zhai B, Xiao L, Piao T and Liu M: Biochanin A inhibits lipopolysaccharide-induced inflammation in human umbilical vein endothelial cells. Life Sci 136: 36-41, 2015.

14. Wu WY, Wu YY, Huang H, He C, Li WZ, Wang HL, Chen HQ and Yin YY: Biochanin A attenuates LPS-induced pro-inflammatory responses and inhibits the activation of the MAPK pathway in BV2 microglial cells. Int J Mol Med 35: 391-398, 2015.

15. Puthli A, Tiwari R and Mishra KP: Biochanin A enhances the radiotoxicity in colon tumor cells in vitro. J Environ Pathol Toxicol Oncol 32: 189-203, 2013.

16. Szliszka E, Czuba ZP, Mertas A, Paradysz A and Krol W: The dietary isoflavone biochanin-A sensitizes prostate cancer cells to TRAIL-induced apoptosis. Urol Oncol 31: 331-342, 2013.

17. Su SJ, Chow NH, Kung ML, Hung TC and Chang KL: Effects of soy isoflavones on apoptosis induction and G2-M arrest in human hepatoma cells involvement of caspase- 3 activation, Bcl-2 and Bcl-XL downregulation, and Cdc2 kinase activity. Nutr Cancer 45: 113-123, 2003.

18. Kim JS, Park MR, Lee SY, Kim DK, Moon SM, Kim CS, Cho SS Yoon G, Im HJ, You JS, et al: Licochalcone A induces apoptosis in KB human oral cancer cells via a caspase-dependent FasL signaling pathway. Oncol Rep 31: 755-762, 2014.

19. Zhang Z, Li HM, Zhou C, Li Q, Ma L, Zhang Z, Sun Y, Wang L, Zhang X, Zhu B, et al: Non-benzoquinone geldanamycin analogs trigger various forms of death in human breast cancer cells. J Exp Clin Cancer Res 35: 149, 2016.

20. Kim SM: Human papilloma virus in oral cancer. J Korean Assoc Oral Maxillofac Surg 42: 327-336, 2016.

21. Seo YS, Yim MJ, Kim BH, Kang KR, Lee SY, Oh JS, You JS, Kim SG, Yu SJ, Lee GJ, et al: Berberine-induced anticancer activities in FaDu head and neck squamous cell carcinoma cells. Oncol Rep 34: 3025-3034, 2015. 
22. Howren MB, Christensen AJ, Hynds Karnell L, Van Liew JR and Funk GF: Influence of pretreatment social support on healthrelated quality of life in head and neck cancer survivors: Results from a prospective study. Head Neck 35: 779-787, 2013.

23. Zafar A, Singh S and Naseem I: Cytotoxic activity of soy phytoestrogen coumestrol against human breast cancer MCF-7 cells: Insights into the molecular mechanism. Food Chem Toxicol 99: 149-161, 2017.

24. Lee GA, Choi KC and Hwang KA: Kaempferol, a phytoestrogen, suppressed triclosan-induced epithelial-mesenchymal transition and metastatic-related behaviors of MCF-7 breast cancer cells. Environ Toxicol Pharmacol 49: 48-57, 2017.

25. Roh T, Kim SW, Moon SH and Nam MJ: Genistein induces apoptosis by down-regulating thioredoxin-1 in human hepatocellular carcinoma SNU-449 cells. Food Chem Toxicol 97: 127-134, 2016.

26. Bhardwaj V, Tadinada SM, Jain A, Sehdev V, Daniels CK, Lai JC and Bhushan A: Biochanin A reduces pancreatic cancer survival and progression. Anticancer Drugs 25: 296-302, 2014.

27. Tsuruo T, Naito M, Tomida A, Fujita N, Mashima T, Sakamoto H and Haga N: Molecular targeting therapy of cancer: Drug resistance, apoptosis and survival signal. Cancer Sci 94: 15-21, 2003.

28. Hassan M, Watari H, AbuAlmaaty A, Ohba Y and Sakuragi N Apoptosis and molecular targeting therapy in cancer. BioMed Res Int 2014: 150845, 2014.

29. Stec R, Bodnar L, Smoter M, Maczewski M and Szczylik C: Metastatic colorectal cancer in the elderly: An overview of the systemic treatment modalities (Review). Oncol Lett 2: 3-11, 2011.

30. Hsieh MJ, Chen JC, Yang WE, Chien SY, Chen MK, Lo YS, Hsi YT, Chuang YC, Lin CC and Yang SF: Dehydroandrographolide inhibits oral cancer cell migration and invasion through NF-кB-, AP-1-, and SP-1-modulated matrix metalloproteinase-2 inhibition. Biochem Pharmacol 130: 10-20, 2017.

31. Huang C, Jacobson $\mathrm{K}$ and Schaller MD: MAP kinases and cell migration. J Cell Sci 117: 4619-4628, 2004

32. Kole L, Giri B, Manna SK, Pal B and Ghosh S: Biochanin-A, an isoflavon, showed anti-proliferative and anti-inflammatory activities through the inhibition of iNOS expression, p38-MAPK and ATF-2 phosphorylation and blocking NF- $\kappa$ B nuclear translocation. Eur J Pharmacol 653: 8-15, 2011.
33. Hsu HH, Liu CJ, Shen CY, Chen YJ, Chen LM, Kuo WH, Lin YM, Chen RJ, Tsai CH, Tsai FJ, et al: p38 $\alpha$ MAPK mediates $17 \beta$-estradiol inhibition of MMP-2 and -9 expression and cell migration in human lovo colon cancer cells. J Cell Physiol 227: 3648-3660, 2012.

34. Fromigué $\mathrm{O}$, Hamidouche $\mathrm{Z}$ and Marie PJ: Blockade of the RhoA-JNK-c-Jun-MMP2 cascade by atorvastatin reduces osteosarcoma cell invasion. J Biol Chem 283: 30549-30556, 2008.

35. Bindhu OS, Ramadas K, Sebastian P and Pillai MR: High expression levels of nuclear factor kappa $\mathrm{B}$ and gelatinases in the tumorigenesis of oral squamous cell carcinoma. Head Neck 28 : 916-925, 2006

36. Qin L, Liao L, Redmond A, Young L, Yuan Y, Chen H, O'Malley BW and $\mathrm{Xu}$ J: The AIB1 oncogene promotes breast cancer metastasis by activation of PEA3-mediated matrix metalloproteinase 2 (MMP2) and MMP9 expression. Mol Cell Biol 28: 5937-5950, 2008.

37. Tang $\mathrm{H}$ and Xue G: Major physiological signaling pathways in the regulation of cell proliferation and survival. Handb Exp Pharmacol: Feb 24, 2017. https://doi.org/10.1007/164_2017_4.

38. Xu Q, Xu HX, Li JP, Wang S, Fu Z, Jia J, Wang L, Zhu ZF, Lu R and Yao Z: Growth differentiation factor 15 induces growth and metastasis of human liver cancer stem-like cells via AKT/ GSK-3 $\beta / \beta$-catenin signaling. Oncotarget 8: 16972-16987, 2017.

39. Tseng HS, Wang YF, Tzeng YM, Chen DR, Liao YF, Chiu HY and Hsieh WT: Aloe-emodin enhances tamoxifen cytotoxicity by suppressing Ras/ERK and PI3K/mTOR in breast cancer cells. Am J Chin Med 45: 337-350, 2017

40. Bahrami A, Khazaei M, Hasanzadeh M, Shahid Sales S, Joudi Mashhad M, Farazestanian M, Sadeghnia HR, Rezayi M, Maftouh M, Hassanian SM, et al: Therapeutic potential of targeting PI3K/AKT pathway in treatment of colorectal cancer: Rational and progress. J Cell Biochem: Feb 23, 2017 (Epub ahead of print). doi: $10.1002 / \mathrm{jcb} .25950$.

41. Lux MP, Fasching PA, Schrauder MG, Hein A, Jud SM, Rauh C and Beckmann MW: The PI3K Pathway: Background and treatment approaches. Breast Care (Basel) 11: 398-404, 2016. 